\title{
The Research of Positioning Measurement Based on Sensor Signal Strength
}

\author{
Mack Jianhua $\mathrm{Du}^{1}$ and Roger Liu ${ }^{2}$ \\ ${ }^{1}$ Shanghai Jianqiao University, Shangahai, China \\ ${ }^{2}$ Honeywell(China)Co., Ltd., Shanghai,China
}

\begin{abstract}
Sensor positioning plays animportant role in wireless sensor network (WSN). Thesensor location information is indispensable to a lot of applications. This paper analyzes the wireless sensor node measuring algorithm and the principle of classification, focuses on analyzing the signal strength based ranging method of RSSI, puts forward anintegration positioning method between theoretical and experience of the signal intensity values. This innovative method extends to most complicated positioning applications.
\end{abstract}

Keywords-sensornode; positioning measurement; Signal strength; Integration positioning method

\section{INTRODUCTION}

With the sensor technology rapidly development, WSN is used in the various industries. WSN consists of many sensors with sensing, computing and communicating functions and forms a self-orgnized wireless network. By the sensor collecting theobject data in monitoring area, WSN processes these data into useful and accurate message and transmits them to the management center. WSN is the new generation network and will produce a profund effect on the human life and work[1].

In the many applications of WSN, the location message of sensor or object monitored is critical necessary.

\section{SENSOR LOCALIZATION TECHNOLOGY}

Generally because of the uncertainty of node postion and important of data center, the sensor node becomes as the key point in the WSN. If adopting GPS method, the sensor positioning cost and complexity greatly increases because of tiny sensor device and crypicity factors. It is trend to positioning by sensor isself[2]. No matter what the positioning method, it must need the known-node to determine unkonwn-node, here the known-node is refer as Anchor Node.

Regarding to the positioning mechanism, there are two types: Range-based methord and Range-free methord[3].

The former needs to measure the distance or angle data between the unknown-node and anchor node, then calculates thenodelocationdataby trilateration measurement, triangle measurement or maximum estimation method. While later only needs the connection of network to determine the position of unknown-node in network.

Range-based method focuses to measure the Receiced Signal Strength Indicator (RSSI), Time of Arived (TOA),Time Deffience of Arived (TDOA), and Angle of Arrived (AOA) etc., then fixes the position by suitable algorithm. This method advantage is more accuracy. However the disadvantages are high quality need for node hardware, bigger power consumption and environment humidity which affects the precision.

Range-free method adopts the hop algorithm and connection of network to fix the node position such as DV-hop algorithm, APTI algorithm, and Amorphous algorithm etc., it does not need any infrastructure like base station and GPS. Above several algorithms, more or less, are with some insufficient factors. In general, the positioning precision and speed of convergence in this method basically depends on the estimation precision on the distance of per hop in the network. It is more suitable for simple topology in network [4]. The mixture using above algorithms realizes the better precision or is lower standard for certain measure data.

\section{INDOOR WIRELESS POSITIONING BASED ON RSSI}

This paper mainly researches the indoor sensor node positioning in WSN. In monitoring area, there are severallocated signal receivers, when the transmitter produces the electromagnetic signal, receivers get the signal and measure its signal strengthas well as electromagneticcharacteristic, and finally the accurate transmitterlocation isdetermined[5].

By the signal strength to fix the location in indoor environment, there are severalnegativefactors such as obstacle, non-sight distance, multipath etc. for position precision. Moreover there is much workload for setting actual measure database. This paper provides an integration positioning method bewteen theoretical and experience in the signal strength values. Meanwhile by combineing the model of signal transmission and measure on site, it not only ensures the precision, but also highly reduces the workload on site. In the simple enviroment without any obstacle, it adopts the theoretical database which is educed from model of signal transmission; in the complexity enviroment with many obstacles, it uses experience database which comes from measure value on site.

\section{A. Building the Theoretical Value Database}

It is critical that setting up the transmisttion model of RF signal because there is big difference of transmission loss in different environment. Hence it needs to build different signal transmission model based on the relevant environment.

1) Choosing the suitable transmission model

When the signal transmitting, there is negative effect from other types of signal such as reflection, refraction, and diffraction etc. The signal strength received is added from all 
signal components, it is very strongin sometime, and weak in other time. From large quantity of engineering practice, the received signal strength obeys "Log-normal rule", its probability model of signal transmission is as following:

$$
p(d)[d B m]=p\left(d_{0}\right)[d B m]-10 n_{p} \lg \left(\frac{d}{d_{0}}\right)+X_{s}
$$

Here, $p(d)$ is signal strength received as distwo nodes distance between sending and receiving; $d_{0}$ is reference distance in two nodes; $p\left(d_{0}\right)$ is signal strength received as $d_{0}$ is distance in two nodes; $n_{p}$ ispath loss; $X_{s}$ obeys average value 0 .Standard difference issGaussian distributionrandom variable, which is indepedent of transmisson distance. Itmeans that loss measured could be quitely diffrent as the same trnsmission distance. $X_{s}$ is used to reduce the deviation of signal strength, that reducs positioning deviation as well.

From large quantity practice, the probability model is better reflecting the signal transmission rule comparing to other model, moreover it is easy to use. This paper chooses the probability model as the basis for theoreticalvalue database.

\section{2) Dividing positioning area}

In the simple square area without any obstical, setting the signal node $\mathrm{M}_{1}, \mathrm{M}_{2}, \mathrm{M}_{3}, \mathrm{M}_{4}$ at the four corners, $\mathrm{m}$ is unit length for dividing area, as show figure 1 . Then the distances from point $\mathrm{A}_{1}\left(\mathrm{x}_{1}+\mathrm{m}, \mathrm{y}_{1}+\mathrm{m}\right)$ to four corners are respectively $\sqrt{2} \mathrm{~m}, \sqrt{26} \mathrm{~m}, 5 \sqrt{2} \mathrm{~m}, \sqrt{26} \mathrm{~m}$. Based on the experience, setting $\mathrm{m}$ length is $2 \sim 4 \mathrm{~m}$,( it depends on the measure precision and real time positioning calculation ).

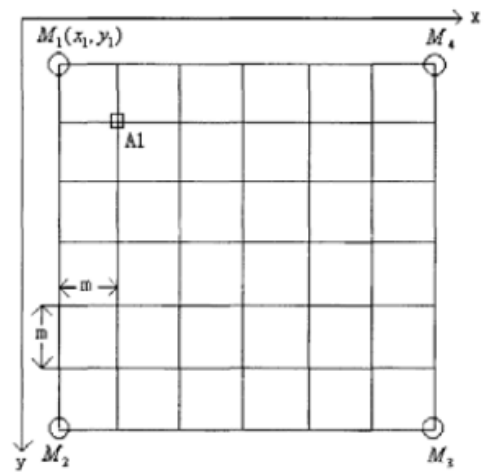

FIGURE I. DIVIDING AREA SHOW.

3) Creating the "location $\sim$ signal strength" relation characteristic

Generally the "Location Signal Strength" Relation Characterstic contains three elements: location coordinate, average value of signal strength , and standard difference of signal strength . There is example for setting point $A_{1}$.

Location coordinate : $\left(\mathrm{x}_{1}+\mathrm{m}, \mathrm{y}_{1}+\mathrm{m}\right)$

Average value of signal strength: by signal transmission model, transferring the four distances from $A_{1}$ to $M_{1}, M_{2}, M_{3}, M_{4}$ (respectively $\sqrt{2} \mathrm{~m}, \sqrt{26} \mathrm{~m}, 5 \sqrt{2} \mathrm{~m}, \sqrt{26} \mathrm{~m}$ ) into average values of signal strength of $\mathrm{P}_{1}, \mathrm{P}_{2}, \mathrm{P}_{3}, \mathrm{P}_{4}$ ( Transfer formula as above (1)).
Standard difference of signal strength : It expresses deviation degree between measure value and average value in signal strength, as below formula:

$$
\int_{1}=\sqrt{\mathrm{E}\left\{\left[\Pi-\Pi_{1}\right]\right\}},(1=1,2,3,4)
$$

$P$ is measure value in signal strength, Eisaverage value. Thus it creates the relation of location coordinate and signal strength between the $\mathrm{A}_{1}$ and each $\mathrm{M}$ point:

$$
\left(x_{1}+m, y_{1}+m\right) \sim\left[\begin{array}{ll}
p_{1} & \sigma_{1} \\
p_{2} & \sigma_{2} \\
p_{3} & \sigma_{3} \\
p_{4} & \sigma_{4}
\end{array}\right]
$$

By the same way, getting the relation of location coordinate and signal strength charactersticin other cross points. Its formula is as below:

$$
(\mathrm{x}, \mathrm{y}) \sim\left[\begin{array}{ll}
\mathrm{SAVG}_{1} & \mathrm{SDEV}_{1} \\
\mathrm{SAVG}_{2} & \mathrm{SDEV}_{2} \\
\mathrm{SAVG}_{3} & \mathrm{SDEV}_{3} \\
\mathrm{SAVG}_{4} & \mathrm{SDEV}_{4}
\end{array}\right]
$$

Here $\mathrm{SAVG}_{\mathrm{i}}$ is average value of signal strength, $\mathrm{SDEV}_{\mathrm{i}}$ is standard difference value, $\mathrm{i}=1,2,3,4$.

Thus it produces the theoretical value database in signal strength.

4) Checking correction theoretical value database

By the actual measurement value checks the correction theoretical value database. It is the concrete method that selects equably several points and measures their signal strength in the positioning area. If the difference is little between actual measuring value and theoretical value in database, there is no correction; otherwise, the actual measure value is used instead of theoretical value in database for the test ponit. Finally it creates one complete theoretical value signal strength database.

\section{B. Building the Experience Value Database}

Because of many walls, bodies, obstacles in indoor, there arevarious other signals such as reflection, refraction, and diffraction etc. The signal strength received is added by all signal components via multipath. Hence the RF signal strength distribution is closely related to the indoor environment. In order to realize the positioning based on signal strength in this complicated environment, it is the feasible method to use experience value database.

There are four beacon nodes in positioning system. The indoor plane graph, beacon node location, and transmitted power of unknown-node are known. The monitoring area is divided into multi units. The sample value of signal strength is collected many times at different location in per unit, and then the average value of signal strength and standard difference value can be calculated. Meanwhile these values are as the basis of experience value database. When the signal strength value measured is near to the certain value in the unit of database, this center location of unit is as unknown-node location. 
The method of building experience database is as below:

\section{1) Dividing the minimumpositioningunit}

The monitoring area is divided into multi units, and the each unit is same, e.g. one space $(1.5 \mathrm{~m} \mathrm{x} 4 \mathrm{~m})$ is as a unit. It is considered on the workload and permissible positioning error. One unit square is limited within $6 \sim 16 \mathrm{~m}^{2}$.

\section{2) Actual measure signal strength value}

In unit 1, one transmitting node with known power is located randomly, beacon node 1 collects signal strength value for 100 times from this transmitting node, Unit 1, corresponding to the beacon node1, gets the vector value of signal strength $\overrightarrow{R S}_{1}=\left(\mathrm{RS}_{1.1}, \mathrm{RS}_{1.2}, \ldots, \mathrm{RS}_{1.100}\right)$. By the same way, unit1 gets vector values corresponding to other three beacon nodes.

\section{3) Getting signal strength characteristic}

From large quantity collections, it is discovered that signal strength is disturbed from white nose $X_{s}$. The white nose is $X$ ${ }_{s} \sim N\left(0, S^{2}\right)$. Thus it is efficient to express the signal strength characteristic by getting the average value and standard difference in signal strength. By $\overrightarrow{R S}_{1}=\left(\mathrm{RS}_{1.1}, \mathrm{RS}_{1.2}, \ldots, \mathrm{RS}_{1.100}\right)$, unit 1 , corresponding to the beacon node1, gets the average value SAVG 1.1 and standard difference $\mathrm{SDEV}_{1,1}$ in signal strength. By the same way, unit1 gets average values $S_{A V G}$ and standard difference $\operatorname{SDEV}_{1 . \mathrm{i}}, \mathrm{i}=(2,3,4)$ in signal strength corresponding to other beacon nodesi $=(2,3,4)$.

4) Building the "location signal strength" relation characteristic

The unit1 (center location coordinate $\left.\left(\mathrm{x}_{1}, \mathrm{y}_{1}\right)\right)$ builds the collected signal strength relation characteristics with each beacon node. Its formula is as below:

$$
\left(\mathrm{x}_{1}, \mathrm{y}_{1}\right) \sim\left[\begin{array}{ll}
\mathrm{SAVG}_{1.1} & \mathrm{SDEV}_{1.1} \\
\mathrm{SAVG}_{2.2} & \mathrm{SDEV}_{2.2} \\
\mathrm{SAVG}_{3.3} & \mathrm{SDEV}_{3.3} \\
\mathrm{SAVG}_{4.4} & \mathrm{SDEV}_{4.4}
\end{array}\right]
$$

Hence the other unit (center location coordinate $\left(x_{j}, y_{j}\right)$ ) in area collected signal strength relation characteristic with other each beacon node is set up as well. Its formula is as below:

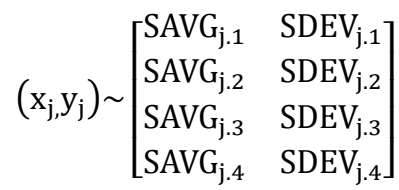

Thus one complete experience value signal strength database sets up.

By combining the theoretical value database and experience value database, that creates one theoretical- experience value database. It is as real time positioning base.

The integrated positioning method of theoretical- experience value database, the advantage is very obvious: when being in complicated environment, multi obstacle area, the experience value database is adopted; when being in simple environment, area without any obstacle, the theoretical value database is used. This method reduces the multipath effect, promotes the positioning precision and decreases the actual workload in the experience value database. Meanwhile it solves the problem which changes the positioning algorithm when monitored object moves from simple area to complicated area.

\section{CONCLUSION}

Today the wireless network covers more and more large area, location message is becoming one of key basic information. Wireless positioning technology rapidly development, the related services is stepping into people 's life. As the basic technology of WSN, this positioning measurement technology based on signal strength is of lower cost, less equipment, ease to use and so on benefits. It is especially suitable for wireless positioning system in low power and cost properties.

The innovation of this paper is integration positioning method by theoretical and experience value database, it covers most application fields based on signal strength.

\section{REFERENCE}

[1] A.Dan, S. Halder, S. Dasbit "Localization with Enhanced Accuracy Location Using RSSI in WSN,” IEEE Inter'l Conference on Advanced Networks \& TelecommunicationSystems, 011,34(17):1-6

[2] Zhou Lijun, Liu Yu."Study on Node Self-Localization Based on RSSI in WSN ” Electronic Measurement Technology, Vol.33, No.8, August,2010

[3] Andy Harter, Andy Hopper. Adistributed location system for the active offiee. IEEE Network, 1994, 8(l):62 - 70.

[4] C.henXijian, Cheng Lianlun."Study and Implementation of Power Match Algorithm Bassed on RSSI," Chinese Journal of Sensor and Actuators Vol.26,No.5, May, 2013

[5] Roy want, Andy Hopper, VernieaFaleao and Jon Gibbons. "The Active Badge Location System," ACM Transsation on Information System January 1992, 10(1):91-102 